\title{
Sobre cómo razonamos La Justicia
}

José María Arredondo Bartolo* Recibido: 04 junio 2016

\section{Resumen}

En este trabajo examinamos la justicia desde el punto de vista del sujeto que razona lo que es justo, desde un punto de vista descriptivo. El reto será conocer los elementos de un razonamiento sobre lo que es justo, exponiendo cuestionamientos a sus bases. Así pues, vamos a describir como razonamos cuando consideramos que algo es justo, y haremos una distinción entre razonamientos morales precipitados y los de carácter deliberativo o lentos. Al final estaremos dispuestos a diferenciar entre como razonamos moralmente de hecho y cómo debemos hacerlo preliminarmente, exponiendo posibles líneas de investigación derivadas de nuestras conclusiones.

Palabras clave: Razonamiento moral, Neurociencia, Convención moral, Razonamiento moral precipitado, Razonamiento moral deliberado.

\section{About how we reason justice}

\begin{abstract}
In this paper we examine justice from the point of view of the person that is reasoning what is fair, from a descriptive point of view. The challenge will be know the elements about a reasoning of what is fair, exposing the questions to its bases. Thus, we describe how we reason when we consider that something is fair, and make a distinction between precipitated moral reasoning and deliberative or slow moral reasoning. At the end we will be willing to differentiate between how we actually reason in a moral way, and how we mustdo it preliminarily, exposing possible lines of research derived from our findings.
\end{abstract}

Key words: Moral reasoning, Neuroscience, Moral mainstream, Hasty moral reasoning, Slow moral reasoning.

*Universidad de Guadalajara. chemaarredondo.3@gmail.com Páginas: 41-62 


\section{Sumario:}

I. Planteamiento. II. Perplejidad en los presupuestos del Razonamiento normativo. III. ¿Cómo funciona el razonamiento moral en el cerebro? IV. Hasty and slow moral reasoning. V. Conclusiones. Bibliografía.

\section{Planteamiento}

Entre quienes nos dedicamos a una ciencia social nos encontramos los que preferimos una visión subjetivista. Nuestra principal característica es que para nosotros el individuo es el objeto (mejor dicho sujeto) de conocimiento desde el cual realizamos nuestras investigaciones. Esto tiene sus razones y consecuencias ${ }^{1}$.

Desde hace algún tiempo que las ciencias sociales se han dedicado al estudio de la conducta del individuo en sí, no a partir de conceptos genéricos como sistema jurídico, para tener una opinión más certera o confiable, quizá predecible no tanto explicativa, estudiando una conducta particular, por ejemplo, en su calidad de "económica" o "jurídica" desde ese punto de vista (Cossío, 2010: 12)².

Lo dicho no es algo nuevo, pero me sirve para dejar en claro premisas básicas que justificarán nuestro trabajo, su actualidad y utilidad reflexiva y práctica.

En cuanto al derecho tenemos que desde mediados del siglo XX una buena parte de la filosofía del derecho dirigió su atención por décadas al estudio del proceso de razonamiento y aplicación del derecho en el personaje del juez (Atienza, 2005: UNAM) ${ }^{3}$.

${ }^{1}$ Nota: se trata de explicar la acción social y sus significaciones, en lugar del estudio de conceptos generalizadores, que tienen la intención de explicar las conductas como partes de estructuras exteriores al sujeto que lo determinan. Pensemos en el siguiente concepto: "Estado Democrático Constitucional de Derecho".

${ }^{2}$ Nota: Antecedente explicativo es el fin del medioevo y comienzo del renacimiento, cuando el hombre se coloca en el centro del conocimiento. También, el fin del siglo XIX y comienzo del XX, particularmente en ciencias como la sociología, la política y el derecho en que se llegaron a desarrollar conceptos tales como "leyes del desarrollo social" o "el espíritu del pueblo", para pasar a estudiar al hombre como persona, siendo éste un punto de partida común en el trabajo de científicos sociales.

${ }^{3}$ Nota: En la obra de Atienza, "Las razones del Derecho”, se exponen en orden de aparición las teorías de la argumentación que se enfocaron en lo que se conoce como lógica de los 
Al respecto, el estudio sobre la manera que en razonamos el derecho, ya sea para conocerlo, manifestarlo en leyes o códigos, y sobre todo para aplicarlo a casos concretos, se ha entendido de diferentes formas. Así, por ejemplo, Hans Kelsen nos aclaró en su primera versión de la Teoría Pura del Derecho (1934) que su pretensión epistemológica era que:

"Con la formulación de la norma fundamental la teoría pura del derecho no pretende en absoluto inaugurar un nuevo método cientifico de la jurisprudencia. Solo pretende hacer patente lo que todos los juristas hacenpor lo general inconscientemente - cuando, para entender su objeto, rechazan el derecho natural como instancia de la que podría derivarse la validez del ordenamiento jurídico positivo a pesar de lo cual conciben el derecho positivo como un ordenamiento válido, no como un mero factum de conexiones psíquicas motivacionales, sino como norma(...) intenta únicamente poner al descubierto - a través del análisis del proceder real-las condiciones lógico-trascendentales del método usual que siempre se ha empleado para el conocimiento del derecho positivo (...)" (Kelsen, 1934:85).

Por su parte, tiempo después Ronald Dworkin escribió sobre la manera en que los jueces razonaban la constitución (norteamericana), y escribió:

"I should make plain first, however, that there is nothing revolutionary about the moral reading in practice. So far as American lawyers and judges follow any coherent strategy of interpreting the Constitution at all, they already use the moral reading, as I hope this book will make plain (... ${ }^{4}$ ”.

juristas para integrarlos en una misma categoría. Se trata de teorías sobre la manera en que se razona en el ámbito juridico (especialmente el judicial) y propuestas sobre cómo se debería de razonar en casos difíciles. Aquellas teorías fueron: Viehweg (1953); Perelman y OlbrechTyteca (1958); Toulmin (1958); MacCormick (1978); y Robert Alexy (1978).

Véase:Atienza, Manuel, Las razones del Derecho, Instituto de Investigaciones Jurídicas UNAM, México, 2007.

${ }^{4}$ T. E. "primero debo hacer una aclaración, que no hay en la práctica nada de revolucionario en la lectura moral. Así que cuando los abogados y jueces norteamericanos siguen cualquier estrategia coherente de interpretación constitucional de hecho, ya usan la lectura moral, así como espero que este libro lo deje claro". 
"So, to repeat, the moral reading is not revolutionary in practice. Lawyers and judges, in their day-to-day-work, instinctively treat the Constitution as expressing abstract moral requirements that can only be applied to concrete cases through fresh moral judgments (... $)^{5 "}$.

Por supuesto que citar a Kelsen y Dworkin para explicar un mismo aspecto del derecho nos impone una pregunta:¿Por qué hacer énfasis en estas citas textuales, más aun tratándose de autores que pueden considerarse diametralmente opuestos en cuanto que pertenecen a tradiciones jurídicas distintas y en que la filosofía de cada uno tenía objetivos que parecería difícil encontrarles aspectos en común?

Seamos concretos. Ambas teorías, aunque muy diferentes, tienen en común el ser esencialmente descriptivistas, antes que tratar, como lo hizo Dworkin, de proponer cual debería ser nuestra postura epistemológica y practica ante el derecho.

Ambas nos quieren decir que no pretendían explicarnos como "debían" razonar jueces o abogados, sino como razonan de hecho. Esto suscita muchas cuestiones a las diversas teorías del derecho en la segunda mitad del siglo $\mathrm{XX}$, al considerarse preliminarmente que no tenían nada en común. ${ }^{6}$

Si el lector está de acuerdo con nosotros entonces podemos proponer la siguiente idea: la teoría del razonamiento jurídico requiere apartarse del estudio del razonamiento que realizan los operadores jurídicos que razonan con normas en un ámbito institucional: el juez o cualquier operador del derecho. Invitamos a llevar nuestro estudio al individuo común, al ser humano

${ }^{5}$ T. E. "Así que, repito, la lectura moral no tiene nada de revolucionaria en la práctica. Abogados y jueces, en su trabajo diario, tratan instintivamente a la Constitución como una expresión abstracta de requerimientos morales que solo pueden ser aplicados a casos concretos a través de renovados razonamientos morales"

${ }^{6}$ Nota: Se habló de variadas categorías teóricas que volvieron confuso el estudio teoríco del derecho, a veces innecesariamente. Tales categorías iban desde las conocidas de "positivismo jurídico", "iusnaturalismo jurídico", post-positivismo jurídico", "neo-iusnaturalismo jurídico" y "positivismo incluyente", entre muchas otras.

Para conocer más acerca del tema véase: Bayón, Juan Carlos.El contenido minimo del positivismo jurídico.Universidad Autónoma de Madrid. Disponible en http//: www.enj. org(consultado el 15 de Febrero del 2016). 
en general, a cualquiera que pueda realizar razonamientos normativos en cualquier circunstancia.

Sin embargo, el tema no es algo tan novedoso, aunque como lo redactamos lo parezca. Los estudios a los que nos referimos se acercan a la neurociencia, que tienen mucho que decir sobre la manera en que razonamos normativamente, y de la cual nos dedicaremos en seguida, no sin advertir nuestras limitaciones, pero que sin embargo esperamos aminorar proponiendo un trabajo que abone a discutir sobre estos temas.

\section{Perplejidad en los presupuestos del Razonamiento Normativo}

Desde hace algún tiempo teóricos del razonamiento normativo nos han convencido de que en general una conclusión prescriptiva, $v$ gr. de tipo moral, requiere de un tiempo justo y una cantidad de información mínima necesaria ${ }^{7}$. Un buen ejemplo de ello es que al juez le llega la información y se toma su tiempo para determinar lo que el derecho prescribe en un caso concreto. Por otro lado, no es difícil atestiguar acciones "buenas" realizadas instantáneamente, y en estos casos algunos podrían pensar que no es tan importante que no se halla deliberado al realizar un salvamento instantáneo en una situación límite o que se crea instantáneamente que el padre que golpea a una de sus hijas en público, excediendo una corrección moderada, hace algo malo.

Sobre lo anterior tenemos algunas preguntas: ¿Por qué en aquellos casos se justifica decir que no es importante, antes de salvar a una persona en una situación límite o calificar de mala una corrección excesiva, que se haya deliberado sobre ello? ¿Por qué no pensar que esas personas pudieron haber hecho o pensado lo contrario, esto es, no salvarla o considerar que las correcciones excesivas son buenas? ¿De dónde proviene ese "paradigma moral automático"? ¿Sigue siendo buena la acción instantánea a pesar de la falta de deliberación, considerando la premisa que supone los razonamientos normativos como integrados por información y tiempo necesario? ¿Podría un juez actuar determinado por ese "paradigma moral automático”? ¿Sería

${ }^{7}$ Vease: Bayón, Juan Carlos, Sobre la racionalidad de dictar y seguir reglas. Revista Doxa, núm. 19,España, Universidad de Alicante, 1996 
irracional, e inaceptable, su sentencia? ¿El derecho podría aceptar decisiones paradigmático-morales como éstas?

Está claro que aquí no encontrara el lector un trabajo intelectual de tipo conceptual, no queremos responder a la pregunta qué es la justicia, que nosotros equiparamos a la otra sobre qué es bueno. Teorías y laberintos tanto racionalistas como metafísicos y teológicos han sido escritos desde hace mucho, y aquello no es a lo que pretendemos dedicarnos; preferimos dejar por ahora las cosas como están ${ }^{8}$.

Por otro lado, nuestro trabajo tampoco trata sobre principios o valores finales que el bien debe tender a lograr, que son objeto también de argumentos que muchas veces son tautológicos por su excesiva obviedad. Lo decimos así porque decir que lo bueno es aquello que nos hace felices es resultado muchas veces de la propia facultad del término a apreciarlo, es decir, es una palabra que causa emociones que muchos estamos dispuestos a traducir en pensamientos sobre lo que siempre hemos deseado tener o sobre la manera en que creemos que debe ser la sociedad. En fin, no es nuestra intención pensar y enroscarnos en esos problemas que para nosotros todavía no tienen solución que nos convenza.

\section{III. ¿Cómo funciona el razonamiento moral en el cerebro?}

Tenemos que asumir una postura previa. Dentro de las varias maneras de entender la relación entre lo mental y lo corporal, en nuestro caso la relación entre el pensamiento y la función cerebral, nos favorecemos con una postura que se conoce como psicobiología9.

Esta postura cree que la conducta es una respuesta a estímulos externos e internos, y que la mente es un conjunto de procesos cerebrales de tipo especial. Además, la psicología tiene como objeto la descripción, explicación, predicción y modificación de procesos conductuales y mentales. En cuanto a su método incluye la observación, la experimentación, modelos matemáticos y estadísticos.

\footnotetext{
${ }^{8} \mathrm{Al}$ respecto véase: Kelsen, Hans.¿Qué es la Justicia? Editorial Fontamara. México.2014.

${ }^{9}$ Vease: Bunge, M; Ardila R. (1988). Filosofía de la psicología. Editorial Siglo XXI:México.
} 
Esta postura considera a la propia psicología como una rama de la biología y las ciencias sociales. Su máxima se resume así: "existo, pienso y me conduzco".

Para ser concretos: ¿Por qué adoptamos ésta postura? Porque es la que asume el reto de identificar los sistemas neurales cuyas funciones específicas son los procesos mentales. En pocas palabras es la actitud científica de la psicología que cree poder conocer que partes del cerebro se activan cuando realizamos un ejercicio mental específico, v.gr., aquel que implica un razonamiento moral. ${ }^{10}$

En consecuencia, la psicobiología une la piscología y la neurociencia. La visión del mundo desde esta unión es un materialismo que no niega la existencia de la ideas, pero si su autonomía, por lo que no pueden ir estas separadas del cerebro. Así pues, sin más preámbulo debemos ir a los datos duros.

How does morality work in the brain? A functional and structural perspective of moral behavior ${ }^{11}$, es el título de un artículo publicado en la revista "Frontiers in Integrative Neuroscience" en 2013, que nos ayuda a entender cuáles son las partes del cerebro relacionadas con las determinaciones conductuales, específicamente las partes que funcionan cuando decidimos que hacer en una situación que implica la moralidad.

En primer lugar, hay en nosotros lo que se conoce como ventromedial prefrontal cortex $\left(\mathrm{VMPFC}^{12}\right)$, y se trata de aquella parte del cerebro que

10 Nota:otras posturas van desde el "animismo" hasta el "materialismo eliminativo". Por ejemplo, la primera postura consideraría que lo mental influye en lo corporal, pero no viceversa; mientras que la segunda considera que nada es mental.

${ }^{11}$ T.E. ¿cómo funciona la moralidad en el cerebro? Una perspectiva funcional y estructural de la conducta moral. Pascual L, Rodríguez P and Gallardo-Pujol D (2013). How does morality work in the brain? A functional and structural perspective of moral behavior Front. Integr. Neurosci. 7:65. doi: 10.3389/fnint.2013.00065. USA. p. 4. (Fecha de consulta: 20 de Enero del 2016) Nota: el estilo de cita en cuanto a la investigación anteriory otras relacionadas con el presente capitulo no sigue EL estilo APA porque es un requisito indispensable prescrito en el texto del propio artículo.

12 Nota: Imaginemos que estamos frente al espejo. El lóbulo frontal se es la parte cubierta por nuestra frente y parte de la primera zona donde tenemos cabello. Se compone de lóbulo frontal derecho e izquierdo. 
tiene una función primordial con respecto a las emociones que de alguna manera influyen en nuestras decisiones morales. No es difícil entender que en ocasiones son las emociones o sentimientos los que nos impulsan más que otra cosa a ayudar a una persona en una situación límite o critica (v.gr. pobreza involuntaria extrema).

Los citados estudios mencionan que las personas que tienen reducidas las facultades de la corteza prefrontal tienden a resolver los dilemas morales de forma utilitaria. Para el caso se cita un conocido dilema que trata sobre el salvamento de cinco personas y el sacrificio necesario de la vida de otra. Entonces, en un lenguaje más coloquial son sujetos a quienes las emociones y los sentimientos no los determinan preponderantemente a la hora de decidir moralmente.

Otra de las características de estas personas es que su capacidad para deducir de forma abstracta las consecuencias de sus actos se ve reducida, por lo que el lóbulo frontal izquierdo se activa más en estas personas cuando presencian la violación de una norma social. Lo traduzco como la dificultad que tienen las personas de percibir con más detalle sucesos que implican injusticias. Lo que llamaríamos insensibilidad.

Así mismo, las personas estamos habituadas al premio y el castigo, y en relación con estos motivadores de la conducta el cerebro cuenta con el orbitofrontal cortex $\left(\mathrm{OFC}^{13}\right)$, bien llamada la función del premio y el castigo, dado que está relacionado con eventos en que estos aparecen. Estaremos de acuerdo que esta parte del cerebro involucra sentimientos como los provocados por el miedo, impulsos efectivos de prejuicios morales.

Ahora bien, las decisiones más influidas por las emociones tienen, sin embargo, un freno, por así decirlo, que mitiga aquel tipo de respuestas. Es lo que opina Joshua Green uno de los investigadores citados en el artículo de Frontiers.

Aquel freno es como la parte racional que controla las emociones, y se conoce con el nombre de dorsolateral prefrontal cortex (DLPFC $\left.{ }^{14}\right)$.Nuestro

${ }^{13}$ Nota: Podemos ubicar esta parte solo debajo del lóbulo frontal.

${ }^{14}$ Nota: lo encontramos un poco por encima del ventromedial prefrontal cortex. 
cerebro distingue en su estructura funcional lo racional y lo que no lo es. Pensemos en una situación en que vemos un hecho considerado injusto: como observador podríamos encontrarnos en el papel de quien puede enjuiciar detenidamente sobre el asunto, a diferencia de los implicados no pueden hacerlo (v.gr.en el robo o asesinato). También podemos pensar en el juez, que tiene conocimiento sobre las normas que califican al hecho como homicidio y su ejercicio es más detenido y racional. En tal caso, los jueces no activan el lado izquierdo del orbitofrontal cortex, dado que no son las emociones o sentimientos los que en este caso deben jugar un papel determinante, sino el dorso lateral prefrontal cortex, es decir, una parte del cerebro más racional.

Ahora bien, a pesar de saber que nuestras decisiones pueden ser tanto emotivas como racionales, no estamos seguros de que el cerebro tenga una zona específica para cada tipo de decisiones, y mucho menos podríamos asegurar que sean totalmente independientes, o que no participen una de otra, pues solo se podría afirmar que:

"The most plausible option is that the "moral brain" does not exist per se: rather, moral processes require the engagement of specific structures of both the "emotional" and the "cognitive" brains, and the difference with respect to other cognitive and emotional processes may lie in the content of these processes, rather than in specific circuits" ${ }^{\prime 5}$.

${ }^{15}$ Ibídem, pp. 5-6

T.E. La opinión más plausible es que "la moral cerebral" no existe por si misma: así que, el procesamiento de la moralidad requiere del encuentro de especificas estructuras, provenientes de ambos campos, lo "emocional" y lo "racional", y la diferencia con respecto a otros procesos emocionales y racionales podría estar en el contenido de esos procesos, más que en los circuitos específicos.

Nota: la traducción de "cognitive” o "cognition” al español es "cognitivo" y "cognición”, respectivamente. El Diccionario del Español de México define "cognoscitivo" de la siguiente manera:

Cognoscitivo:adj. Que es propio o característico de las facultades humanas que llevan al conocimiento de las cosas: "Los sentidos no son exclusivamente de conocimiento (sensibilidad); su función cognoscitiva está mezclada con su función estimulante de los apetitos (sensualidad)".

Por su parte la Real Academia Española define "cognición” así:

Cognición: $f$. conocimiento (\| acción de conocer). 
Entonces, no hay una única estructura y una exclusiva función determinante para el razonamiento moral a nivel cerebral, aunque si se localizan partes del cerebro más relacionadas con las emociones y otras con lo racional, las que se compiten o se relacionan a la hora en que se determina la actitud del sujeto ante un dilema.

Entre la variedad de estructuras y funciones encontramos aquellas donde se inician las percepciones sociales (lóbulo temporal), las estructuras que se activan más cuando se trata de dilemas asociados al concepto de justicia (temporal superior sulcus) y otras que se relacionan con la memoria (lóbulo parietal).

Sepamos también que el cerebro funciona para predecir, tal vez sería mejor decir intuir, la magnitud de las penas impuestas en asuntos criminales (lóbulo límbico), que también se relacionan con situaciones de incertidumbre y alertas en contexto de inequidad, enojo e indignación (corteza insular).

Por otro lado, también hay interesantes funciones del cerebro que ayudan a procesar las reacciones emocionales apropiadas, tales como reír cuando la situación que se vive es de felicidad, o la contraria, de tristeza, cuando el contexto es doloroso o insatisfactorio (hippocampus).

Por último, también tenemos la parte del cerebro que ayuda y funciona cuando aprendemos moralidad y cuando evaluamos un juicio moral (amigdala). Así como aquella parte que se activa cuando realizamos una contribución caritativa (septum).

Al final de cuentas, lo más razonable es coincidir en que cuando pensamos en la relación entre moral y neurociencia no hay más que decir que ésta ofrece una variedad de factores que explican el razonamiento moral. Esto ha llevado a opiniones distintas con respecto a cuál de las dos funciones y estructuras generales determinan más nuestros juicios morales, si es lo racional o lo emocional.

Nota: Consideraremos lo cognitivo como lo racional, aunque los sentidos también tengan la capacidad de conocer, pero de una forma distinta a la de la razón, que toma información no solo de los sentidos, y necesita de un tiempo para emitir una resolución. 
Una postura sobre los anterior lo es "In bad taste: Evidencefor the Oral Origins of Moral disgust"'16, artículo publicado en 2009, en el que se adopta una postura que considera el razonamiento moral como influido, más que por un razonamiento consciente, por nuestra parte emocional o sensitiva.

En tal caso, se ha considerado que ante la violación de normas morales las personas presentan sensaciones de repulsión o disgusto, sean víctimas o testigos de un hecho injusto.

La investigación de la universidad de Toronto se soporta en el estudio del sistema de alerta contra toxinas o de sabores indeseables, en épocas primitivas, que se fue desarrollando hasta que los seres humanos logramos la capacidad, en un plano socio-cultural, de reaccionar contra transgresiones morales o contextos de injusticia.

Sin embargo, los investigadores previenen quela relación entre el efecto en la gesticulación del mentón y los movimientos bucales, por el mal sabor, y el disgusto moral es solo una metáfora, una manera en que podemos explicar la actitud de repulsión ante las injusticias. Entre otras cosas, con ello niegan que en el disgusto oral tenga su origen el disgusto moral.

En la investigación citada, se incluyeron participantes que fueron puestos frente a fotografías con distintas evocaciones conceptuales: tristeza, injusticias, contaminación, que resultaron en una mayor activación de la zona oral y nasal asociada con el gesto común conocido como del mal sabor.

Además, los asistentes estuvieron frente a frente en un programa que les presentaba alternativas de reparto de $\$ 10$ (v.gr. 5-5; 6-4; 9-1; etc.), pero debían tomar en cuenta los participantes que si aceptaban se daba el reparto, sino lo hacían no recibían algo ninguno de ellos, por lo que hubo mayor índice de

${ }^{16}$ T. E. Mal sabor: evidencia sobre el origen oral del disgusto moral.

Chapman, H.A.; Kim, D.A.; Susskind, J.M.; Anderson, A.K. (2009). In bad taste: Evidence for the Oral Origins of Moral disgust. En revista "Science", núm. 323. Toronto: science magazine.

(Fecha de consulta: 20 de Enero del 2016)

Nota: en la anterior cita no se sigue el estilo APA porque es indispensable para poder citar la anterior investigación seguir el formato que en la misma los autores dejaron indicado. 
gesticulación en repartos más injustos que en relación con otros. La pregunta clave en el presente estudio fue: ¿determinan las emociones negativas las decisiones sobre ofertas injustas? Es decir, ¿el sentimiento que nos causa una situación límite o una corrección excesiva determina que consideremos que son injustas? Los investigadores de la universidad de Toronto concluyen que solo tratándose de la sensación de disgusto, conocida como mal sabor o de sabores desagradables, éste si determino en los experimentos las decisiones de rechazo a las ofertas injustas en el ejercicio de reparto. Sin embargo, no sucedió lo mismo con sentimientos como el enojo, el desprecio o la tristeza.

Lo más importante para nosotros es una de las conclusiones a la que llegaron en éste estudio, y que nos indica que:

"Although these correlations cannot establish a causal relationship between decision-making and emotionality, they do suggest that emotions evoked in response to unfairness - in particular disgust - are relevant to subsequent behavioral choices" ${ }^{17}$.

Sin problemas de claridad, se ha entendido que las emociones son más determinantes en decisiones subsecuentes, esto es, en situaciones del presente similares a otras del pasado en las que se presentaron emociones, hayan sido positivas o negativas, y que nos determinan para calificar moralmente una vivencia del presente; es decir, es posible que estemos predispuestos por emociones del pasado a creer que algo es injusto porque alguna vez eso nos disgustó, es decir, nos provocó un mal sabor de boca. Volveremos a ésta idea en el último capítulo del artículo y en nuestra conclusión.

\section{Hasty and slow moral reasoning ${ }^{18}$}

El nombre de este capítulo alude a lo que se conoce como teoría dual del procesamiento de los juicios morales. La teoría dual explica que cuando vemos

${ }^{17}$ Ibidem, pp. 1225.

T. E. Sin embargo, ésta correlación no puede establecer una relación causal entre realizar una decisión y las emociones, ello sugiere que las respuestas que evocan las emociones a injusticias- en particular disgustos- son relevantes para subsecuentes decisiones de conducta. ${ }^{18}$ T. E. "El precipitado y el deliberado razonamiento moral".

Nota: utilizamos las palabras "hasty" y "slow" porque son las más adecuadas para explicar 
o escuchamos una historia en la que puedan ser utilizados los calificativos de bueno o malo, automáticamente tenemos impresiones sensibles de aprobación o desaprobación:

"But without any feeling of having gone through steps of searching, weighing evidence, or inferring a conclusion" $"$.

Así, con la impresión de aprobación podríamos automáticamente salvar a una persona en una situación límite, y también automáticamente podríamos considerar que una corrección excesiva no es buena. Esto nos lleva al concepto de pre-juicios, que, aunque se utiliza en el estudio citado, no lo creemos el más adecuado, porque, como lo veremos más adelante, a pesar de que aquellas impresiones automáticas vienen determinadas por los precedentes emotivos que se fueron arraigando en el "hasty moral reasoning", en realidad siempre hay un juicio previo, es decir, la parte racional podría ser lógicamente siempre previa y determinante.

Lo importante es que ahora sabemos que existen dos tipos de razonamiento moral, y que dependiendo de la situación particular, a veces razonamos problemas morales de forma intuitiva o "no-deliberada", y también hay contextos en que tenemos la oportunidad de tratarlos con detenimiento, con lo que estamos cumpliendo con los dos presupuestos del razonamiento normativo, a saber: tiempo e información justa.

Sin embargo, todavía nos queda responder una por una las preguntas que nos hicimos al principio del artículo, cosa importante porque la primera de ellas constituye nuestra hipótesis, y las demás que nos hicimos derivados

el fenómeno a que aludimos. En el Diccionario Merriam-Websterde Sinónimos y Antónimos en Inglés se entiende por éstas palabras lo siguiente:

Hasty.- Connota prisa y precipitación, y connota con frecuencia el descuido y la confusión o ineficiencia resultante $<$ a hastyinspectionfailed to discover the problem $>$.

Slow.- Empleado a menudo con up o down, también indica una reducción de velocidad, a menudo con intención deliberada $<$ the engineerslowed the train $>$

${ }^{19}$ Ver: Green, Joshua; Haidt, Jonathan, revista TRENDS in cognitive "Science", Vol. 6 núm. 12. USA, 2002, Pp. 96-147. (fecha de consulta: 20 de Enero del 2016)

T.E. Pero sin ninguna sensación de haber pasado a través de etapas de búsqueda, valoración de pruebas o por alguna inferencia concluyente. 
importantes. Pero, primero necesitamos contestar otra cuestión:¿Qué es primero el razonamiento intuitivo o el deliberado?

Esta interrogante se puede problematizar si ponemos atención al momento en que estamos en una situación límite y no tenemos mucho tiempo e información para deliberar, instante en el que como lo hemos venido explicando aparece una intuición moral, que tiene que provenir de alguna parte.

La investigación que hemos citado y en la que se consideró que en estas ocasiones nos vemos determinados por nuestra parte emotiva hace creer que invocamos casos del pasado en que nos encontrábamos en una situación similar a la del presente y entonces reaccionan todas aquellas sensaciones. Entonces, así se explica que tenemos una postura moral para el caso en que ver a un padre ejercer una corrección excesiva nos provoca una "sensación" de repulsión, rechazo, encono, y es por lo cual que creemos que eso "no debe" hacerse, porque es moralmente malo.

Sin embargo, aunque las explicaciones que nos da la neurociencia nos muestran que es nuestra parte emotiva la que reacciona en estos casos, somos de la opinión de que no es el fondo del origen del razonamiento intuitivo moral.

En una situación precipitada es verdad que no queda más que tomar una decisión rápida, invocando nuestras experiencias pasadas, es decir, no tenemos la oportunidad de realizar razonamientos morales novedosos, construir premisas distintas a las que conocemos, justificar una vez más nuestras propias decisiones. Lo anterior, a pesar de que es cierto, ello no impide que después de una decisión moral intuitiva tratemos de justificarla; por supuesto que cuando ya podemos realizarlo con tiempo y más información que la que teníamos en el momento en que se presentó el problema.

Traer del pasado una resolución moral, implica que esta se ha guardado como un paradigma moral, y que constantemente, en el transcurso de nuestra vida personal, las vamos construyendo: premisas, razones, deducciones, una 
lógica, toda una construcción argumentativa que define nuestra postura de vida ante problemas morales. En el ámbito del derecho esto se relaciona conlacoherencia, consistencia y el principio de universalidad aplicado a las decisiones.

Pues bien, la postura es que a pesar de que existan los "hasty moral reasoning" estos también surgieron, poco a poco, en no igual profundidad en todos los casos, de un "slow moral reasoning".

Ahora bien, nuestra hipótesis es la siguiente: ¿Por qué en aquellos casos se justifica decir que no es importante que, antes de salvar a una persona en una situación límite o calificar de mala una corrección excesiva, no se haya deliberado sobre ello? Porque sabemos que ante una situación límite $o$ en un contexto que no permite adquirir información externa y un tiempo determinado para decidir, reacciona en nosotros nuestra parte paradigmáticointuitiva, con lo que calificamos aquellas situaciones como buenas o malas de acuerdo a nuestros precedentes, que se justifican en una postura moral que poco a poco hemos construido cuestionándonos, mucho o poco, sobre un problema moral en particular.

¿Por qué no pensar que esas personas pudieron haber hecho o pensado lo contrario, esto es, no salvarla o considerar que las correcciones excesivas son buenas? Esto es posible, dependiendo del paradigma moral de aquellas personas. Por ejemplo, la cuestión sobre qué pasaría si en una situación límite quien necesitara ser asistido fuese un delincuente que hubiera cometido actos indebidos, nuestra respuesta seria que las personas valorarían las faltas del delincuente y las pondrían en una balanza con el valor de la vida, esto es, previsiblemente, estaríamos ante un ejercicio de ponderación, el cual es un razonamiento muy difundido tratándose de dilemas morales. Así pues, somos de la idea de que pocas personas dejarían de salvar a un ladrón de cartera de ser linchado por un grupo de personas. En cambio el delincuente, antes de serlo, a "pre-meditado" bastante sobre los actos tan particulares a los que decidió dedicarse. En derecho la teoría de la pena justifica de alguna manera los castigos con esta clase de argumentos. 
¿Sigue siendo buena la acción instantánea a pesar de la falta de deliberación, considerando la premisa que supone los razonamientos normativos como integrados por información y tiempo necesario? La pregunta nos exige responder si creemos que una acción moral existe solo si previamente realizamos un juicio.

Ya quedo claro en el desarrollo del trabajo que siempre hay un tipo de razonamiento, aunque sea intuitivo, pero que siempre será al fin de cuentas el resultado de una justificación que se va construyendo y guardando como paradigmas morales. Es decir, no hay acción moral sin razonamiento previo, de hecho no lo hay, pero eso significa que falta por establecer como deberíamos razonar moralmente.

Por otro lado, en una situación para la que no sea necesario un "hasty moral reasoning" es nuestro deber aplicar un "slow moral reasoning". Ello implica ser conscientes y aceptar reglas sobre la deliberación racional, criterios de análisis y evaluación de los argumentos, tales como el acuerdo sobre premisas básicas (valores o principios), estructuras e inferencias lógicas aceptables o una tipología de argumentos justificados (v.gr. analógicos, gramaticales, consecuencialistas, históricos, etc.). Lo anterior cumpliría, sabemos que parcialmente, con algunos presupuestos sobre cómo debe ser un razonamiento moral, sobre todo ante situaciones novedosas y relevantes, v gr. ¿Las parejas del mismo sexo deberían tener el derecho de adoptar como las heterosexuales?

¿Podría un juez actuar determinado por ese "paradigma moral”? De hecho lo hace, porque es evidente que se trata de un personaje que, sin lugar a dudas, ha estado tan involucrado con la ética pública, que no es difícil imaginarlo sentenciando en casos comunes como si se tratara de aplicar un "criterio del juzgado".

A pesar de ello, una razón en contra de esta práctica seria que aunque la mayor parte de los razonamientos que emite un juez sean de este tipo, no significa que así deban ser, y que aceptar el valor de la universalidad, que implica respuestas equivalentes para casos similares, no autoriza al juez 
para omitir un razonamiento particular para cada caso, como si ello fuera una regla de justicia formal. Sin embargo, el juez que aplica constantemente una solución similar a casos fáciles o habituales, no está omitiendo un razonamiento particular para cada caso, sino que aplica una cierta interpretación estándar del derecho, no del caso, porque estos nunca son los mismos. Al juez no se le puede pedir que no forme una interpretación estándar del derecho, porque ello también implica los valores de la universalidad y la coherencia de las decisiones.

¿Sería irracional e inaceptable su sentencia? Creo que esta pregunta puede tener un largo desarrollo, que nos llevaría a repetir muchos de los párrafos que aquí hemos ido escribiendo, por lo que dejamos que el lector confirme si en nuestro trabajo hemos o no convencido de que en ninguna forma seria irracional, y tampoco inaceptable de acuerdo a la regla de justicia formal que dice: "el juez tiene el deber de emitir un razonamiento particular para cada caso".

Por otro lado, el juez si tiene el deber de tratar casos no habituales considerando que en ese tipo de casos podrían resultar derrotables valores y principios como la coherencia o consistencia, la legalidad o la certeza jurídica. Por ejemplo, un juez podría llegar a la conclusión de que se debe sobreponer un tipo de interés individual como el desarrollo de la personalidad para la justificación de la despenalización y regularización del consumo de marihuana.

¿El derecho podría aceptar decisiones paradigmático-morales como éstas? El derecho debe aceptarlas en casos que se consideren habituales y que no impliquen el cuestionamiento de interpretaciones estándar del derecho. Recordemos que en el derecho, desde la época clásica de la jurisprudencia romana hasta la actualidad, se guardaban las decisiones relevantes para que fueran utilizadas como referencias en nuevos casos, y que se la ha conocido como la teoría del "stare decisis", o de la autoridad del precedente.

Los anteriores párrafos en el fondo dan cuenta de que lo único que hemos venido a decir es que razonamos el derecho como "de hecho" razonamos 
normativamente, pero que también podríamos discutir y aplicar ideas sobre la manera en que creemos que "deberíamos" realizar razonamientos morales relevantes.

\section{Conclusiones}

Sin lugar a dudas el que una persona dedicada a los ámbitos profesionales en que se razone con contenido moral no desarrolle la actitud y capacidad para realizar un "slow moral reasoning" implica que la fuente de sus decisiones sean los precedentes, a los cuales se adherirá y defenderá porque es una posición que brinda algún tipo de certeza y seguridad.

No obstante lo anterior, lo dicho no es suficiente. Para establecer bases sobre como deberíamos razonar, los siguientes cuatro elementos preliminares de un razonamiento moral deben tomarse en cuenta:

1) de hecho cuando las personas razonamos moralmente nos vemos influidos tanto por nuestra parte racional como emocional. Ambas juegan un papel importante a la hora de decidir sobre lo que es justo o bueno.

2) De hecho las personas tomamos en cuenta los precedentes, y en estos casos las emociones son más determinantes en decisiones subsecuentes. Esto es, tratándose del valor de la universalidad, justificado racionalmente, las emociones pesarían más.

3) De hecho las personas asumimos interpretaciones estándar de enunciados normativos (valores, principios, reglas, derechos, etc.)

4) De hecho las personas tenemos la capacidad de cuestionar todo lo que implique los anteriores presupuestos.

En consecuencia, un razonamiento moral deliberado debe suponer que el sujeto que lo realiza esta dispuesto a cuestionar su propia moralidad, traducido ello en hacer, sino lo opuesto, si lo que va en sentido diverso de las premisas que acabamos de señalar. Uno por uno nosotros creemos que podría ser de la siguiente manera: 
1) A pesar de que tomar una decisión moral involucra tanto nuestra racionalidad como nuestra sensibilidad, el orden de complementariedad seria que tomáramos en consideración que poseemos sentimientos de confianza y seguridad cuando legitimamos decisiones y formas de poder dando razones a la hora de la deliberación moral, aunque podamos decir que la razón práctica tiene sus límites y problemas a la hora de prescribir. Por su parte, a la sensibilidad debe dejársele también un papel de tipo predominantemente psicológico que conocemos como convicción o adherencia a ciertas ideas morales, que juegan el importante papel de motivar la voluntad de las personas para realizar aquello que consideran debe hacerse.

2) Si de hecho decidir en base a precedentes da lugar a un más importante papel de la sensibilidad, los juicios morales no se justifican solo porque estamos de acuerdo en que nos produce aversión cierto tipo de problemas que hemos conocido en el pasado, sino que debemos justificar que esos acontecimientos son similares o se pueden ubicar en una clasificación común con asuntos del presente. Argumentos como a pari, analógico (por similitudes), a simili o histórico (coherencia y progresividad), entre otros, son comunes cuando se trata de racionalizar una decisión basada en un problema que le antecede. Sin embargo, pocas personas considerarían que un argumento que justifique algún tipo de discriminación racial debe seguir prevaleciendo solo por el hecho de que así se venía decidiendo, y no se desaprueba que una corte suprema decida en sentido diverso de los precedentes.

3) El hecho de las interpretaciones estándar viene dado con lo anterior. Los precedentes son una necesidad, de lo contrario todas las controversias serian casos difíciles. De la misma forma, las interpretaciones estándar de nuestros enunciados básicos, por ejemplo: "todos los seres humanos merecen un mínimo igual de respeto a su integridad personal", es necesario para generar una vida moral en comunidad, y seguridad y confianza en lo que podemos esperar de los demás. Aun así, ante interpretaciones estándar, todos los casos fáciles en los que no se someta a discusión un principio 
deben, implícitamente, justificar que la solución a un caso particular no requiere de un "juicio valorativode excepción o cambio" que explique y justifique decisiones distintas a las que hemos dado a casos similares en el pasado. Por ejemplo: que el mínimo de respeto igual a la integridad de los seres humanos no solo implica abstenerse de lesionar físicamente a cualquier persona, sino también el respeto a su integridad moral y espiritual, o su derecho a la no intervención en su vida privada o intima por parte del Estado o cualquier persona sino es con autorización previa. Incluso, si vamos un poco más lejos, podríamos considerar que el mínimo igual de respeto también aplica para los animales o el entorno natural, con lo que justificamos una pena por daño ecológico o ambiental.

4) Finalmente, que de hecho seamos capaces de cuestionar como lo hicimos en los tres puntos anteriores, no quiere decir que siempre se haga así. Racionalizar la moral en la vida diaria e institucional solo es posible si asumimos esa responsabilidad. De lo contrario, lo bueno o justo se definirá como aquello que tiene más historia, como si contraer matrimonio monógamo o heterosexual fuera bueno o justo solo porque llevamos mucho tiempo haciéndolo.

Algunas cuestiones que podrían abrirse después de lo expuesto son preguntas como:¿las personas tienen derecho a decidir cómo y cuándo morir? ¿Deben ser las personas de distinto sexo cuando pretenden contraer matrimonio civil y vivir en familia? ¿Qué significa respetar la dignidad humana de las demás personas? ¿Es bueno privar de la vida a otros porque no comparten "la religión verdadera" que pregonan otras? ¿Los hombres deben ser juzgados con más severidad que las mujeres por delitos que impliquen el sexo como determinante de la comisión del ilícito?

Sin duda, en la actualidad estamos ante problemas morales muy serios y complejos: la justificación moral de la guerra santa islámica; el trato jurídico del consumo de drogas en México; la dignidad humana y los derechos humanos como una concepción moral sobre la vida; el amor y vida en familia de parejas del mismo sexo; la violencia contra las mujeres y la educación de 
los niños y jóvenes con respecto al sexo opuesto; la diversidad religiosa y de creencias; delitos cometidos por niños y adolescentes y su correspondiente trato penal; los datos personales y la vida privada e íntima en esta época de las tecnologías de la información y la comunicación, el daño moral y las redes sociales, etc.

En fin, la justicia es un reto de todos los días, en todos los ámbitos, donde las convicciones sobre lo que creemos que es bueno deben ser puestas en el banquillo de la inspección constante, porque al final de cuentas en el día a día de cada uno de nosotros, en todos los lugares a los que asistimos, no es difícil darse cuenta que ponemos en práctica una concepción del bien. Tal vez así logremos conocer nuestras convicciones morales más profundas, nuestros principios y valores más arraigados, muchas veces desconocidos por nosotros mismos, que podrían incluso resonar en el absurdo.

Empezar por ello es un buen primer paso para conocer nuestra propia concepción de la justicia, tópico útil cuando se trata de justificar y legitimar la reciprocidad de nuestras relaciones con el poder público e incluso nuestras propias relaciones en el interior de la sociedad civil. Todo ello redundaría en una prevalencia de la razón contra cualquier otra forma de llegar a conclusiones sobre lo que es justo.

Atienza, Manuel, Las razones del Derecho, Instituto de Investigaciones Jurídicas UNAM, México, 2007.

Bayón, Juan Carlos, El contenido mínimo del positivismo jurídico, Universidad Autónoma de Madrid. Disponible en http//: www.enj.org (consultado el 15 de Febrero del 2016).

Bayón, Juan Carlos, Sobre la racionalidad de dictar y seguir reglas. Revista Doxa, núm. 19, España, Universidad de Alicante, 1996.

Bunge, M; Ardila R., Filosofía de la psicología, Editorial Siglo XXI, México, 1988. 


\section{Bibliografía}

Constitución Política de los Estados Unidos Mexicanos. (Consultado el 30 de Enero del 2016). Disponible en: http:// www.diputados.gob.mx

Cossío Díaz, José Ramón, Derecho y Análisis económico, México, FCE, ITAM, 2010.

Chapman, H.A.; Kim, D.A.; Susskind, J.M.; Anderson, A.K. (2009). In bad taste: Evidence for the Oral Origins of Moral disgust. En revista "Science", núm. 323. Toronto: science magazine.

Diccionario del español de México. México. (Consultado el día 01 de febrero del 2016). Disponible en http://dem.colmex.mx/

Diccionario de la lengua española. España. (Consultado el día 01 de febrero del 2016). Disponible en http://dle.rae.es/

Dworkin, Ronald, The moral Reading of the American Constitution, Oxford University Press Inc., New York, United States, 2005.

Green, Joshua; Haidt, Jonathan, revista TRENDS in cognitive "Science", Vol. 6 núm. 12. USA, 2002, Pp. 96-147. (fecha de consulta: 20 de Enero del 2016)

Kelsen, Hans, Teoría Pura del Derecho, Introducción a los problemas de la ciencia jurídica (primera edición de 1934), Madrid, Editorial Trotta, 2011.

Kelsen, Hans, ¿Qué es la Justicia?, Editorial Fontamara, México, 2014.

Merriam-Webster, Incorporated, Diccionario Merriam-Webster de Sonónimos y Antónimos en Inglés, Springfield, Massachusetts, EEUU, 2006.

Pascual L, Rodríguez P and Gallardo-Pujol D (2013). How does morality work in the brain? A functional and structural perspective of moral behavior Front. Integr. Neurosci. 7:65. doi: 10.3389/fnint.2013.00065. USA. p. 4. 